ESJ Humanities

\title{
Dissensions internes du peuple n'zima kotoko et pratique de l'abissa a grand-bassam dans le sud de la Côte d'Ivoire
}

\author{
Arsène Kadjo \\ Maître-assistant, Université Peleforo Gon Coulibaly de Korhogo \\ Jean-Baptiste Blay Anoh Ezan \\ Etudiant en Master 2, Université Peleforo Gon Coulibaly de Korhogo
}

Doi: $10.19044 /$ esj.2021.v17n2p117

Submitted: 27 November 2020

Accepted: 16 December 2020

Published: 31 January 2021
Copyright 2021 Author(s)

Under Creative Commons BY-NC-ND

4.0 OPEN ACCESS

Cite As:

Kadjo A. \& Ezan B.A.J.B. (2021). Dissensions internes du peuple n'zima kotoko et pratique de l'abissa a grand-bassam dans le sud de la Côte d'Ivoire. European Scientific Journal, ESJ, 17(2), 117. https://doi.org/10.19044/esj.2021.v17n2p117

\section{Résumé}

Institution traditionnelle de cohésion sociale, l'Abissa représente pour la société N'Zima le socle des valeurs. Il marque le début du nouvel an consacré à la critique sociale, à la purification et au renforcement des liens communautaires et sociaux. Cependant, fort des dissensions internes qui apparaissent en 2018, une rupture dans sa pratique s'est observée. Le présent article se propose alors de comprendre comment les dissensions internes du peuple N'zima affectent la pratique de l'Abissa ainsi que les conséquences qui en découlent. Pour ce faire, l'entretien et l'observation ont été mobilisés comme techniques. L'enquête a mis en lumière l'intrusion des tiers dans la pratique de l'Abissa et les dissensions politiques comme facteurs de rupture. En effet, l'interventionnisme des pouvoirs dans la fixation des dates des festivités de l'Abissa sont interprétées par une frange ultra-conservatrice de la population comme un acte de désacralisation. A ce facteur, s'ajoute la position politique jugée partisane du souverain N'zima qui a exacerbé des tensions sociales et communautaires déjà assez fortes dans un contexte électoral cristallisé par la violence. Comme conséquences de cette situation confligène, la profanation de l'Abissa avec son corollaire la perturbation des énergies cosmiques en faveur du peuple, de même que la fragilisation des relations sociales se soldant par la récurrence des conflits intercommunautaires. La compréhension du phénomène s'est faite à partir la théorie des parties prenantes (J.D. Rendtoff, M. Bonnafous-Boucher, 2014) qui permet à partir 
de leur positionnement dans les actions d'identifier les acteurs en confrontation et les logiques de leurs actions.

Mots clés : Abissa, contestation, dissension, désacralisation, Grand-Bassam

\title{
Internal Dissensions of the N'zima Kotoko People and the Practice of Abissa in Grand-Bassam in the South of Côte d'Ivoire
}

\author{
Arsène Kadjo \\ Maître-assistant, Université Peleforo Gon Coulibaly de Korhogo \\ Jean-Baptiste Blay Anoh Ezan \\ Etudiant en Master 2, Université Peleforo Gon Coulibaly de Korhogo
}

\begin{abstract}
As a traditional institution of social cohesion, Abissa represents the foundation of N'Zima society's values. It marks the beginning of the new year devoted to social criticism, purification and the strengthening of community and social ties. However, with the internal dissensions that will appear in 2018, a break in its practice has been observed. This article therefore sets out to understand how the internal dissensions of the N'zima people affect the practice of Abissa and the consequences that follow. In order to do so, interviewing and observation were used as techniques. The investigation highlighted the intrusion of third parties in the practice of Abissa and political dissension as factors of rupture. Indeed, the interventionism of the powers that be in setting the dates of Abissa festivities is interpreted by an ultraconservative fringe of the population as an act of desacralization. In addition to this factor, the political position of the sovereign N'zima, considered to be partisan, has exacerbated social and community tensions which are already quite high in an electoral context crystallised by violence. The consequences of this conflict situation are the desecration of Abissa with its corollary, the disruption of cosmic energies in favour of the people, as well as the weakening of social relations resulting in the recurrence of inter-community conflicts. The understanding of the phenomenon is based on the theory of stakeholders (J.D. Rendtoff, M. Bonnafous-Boucher, 2014), which makes it possible to identify the actors in confrontation and the logics of their actions on the basis of their positioning in the actions.
\end{abstract}

Keywords: Abissa, protest, dissension, desacralization, Grand-Bassam 


\section{Introduction}

S'interroger sur la relation entre la politique et les institutions traditionnelles notamment de cohésion sociale suppose que l'on prenne en compte l'articulation très étroite entre les modes de construction et de codification des dernières citées dans un contexte sociopolitique surtout africain en perpétuelle mutation. Les enjeux nouveaux dans les échéances électorales affectent très souvent la stabilité sociopolitique des sociétés (E. Benn et al., 2010, p 502). La régulation des pratiques et la distribution des ressources matérielles et de l'autorité s'en trouvent alors fortement perturbées (G. Balandier, 1960, p 10). Ainsi, la problématique de la survivance des institutions stables par nature et par fonction (D. Paulme, 1960, p 21) se pose avec acuité dans les sociétés africaines modernes. Il en va ainsi de la société N'zima, creuset de la pratique de l'Abissa, une institution sociale traditionnelle de cohésion sociale. Caractérisée comme l'une des institutions traditionnelles fortes en Côte d'Ivoire à l'instar du Dipri du peuple Abidji (R. K. Allou, 2013, p. 178), celle-ci connaîtra dans son processus de pérennisation une rupture mettant ainsi en mal sa notoriété jusqu'alors «inviolable. »à Grand-Bassam, la métropole médiévale N'zima (D. N'doli, 2014, p. 18 ; L. A. Kouamé, Sdnl, p. 5).

Les dernières échéances électorales couplées des conseillers régionaux et des conseillers municipaux du 13 octobre 2018 (CEI, 2018) ont vu en effet apparaître dans l'arène sociale N'zima un antagonisme. Il met aux prises les tenants du pouvoir traditionnel incarné par le souverain à qui il est reproché son immiscion dans le jeu électoral et son soutien ouvert au candidat du RHDP. Ce grief est porté par une frange de la population essentiellement composée des jeunes qui s'identifient au candidat adverse à celui soutenu par le roi $^{1}$ qui lui reproche sa partialité. Il s'en est suivi des affrontements à la proclamation des résultats à l'issue du premier et du second tour qui donnaient vainqueur le candidat du RHDP et ce, suite aux contestations du candidat du PDCI et de ses partisans.

Les velléités contre le roi et autres contestations qui s'en suivirent vont révéler l'équilibre précaire constamment menacé par le surgissement de contradictions nouvelles qui provoquent des crises plus ou moins profondes (M. Gueye, 1983, p. 1). Les plus en verve dans ce contexte N'zima précaire sont les jeunes qui s'en prirent aux symboles les plus sacrés de leur communauté. La coïncidence des élections notamment le second tour à la

\footnotetext{
${ }^{1}$ A Grand Bassam, deux candidats étaient déclarés : il s'agit de monsieur Jean Louis MOULO, Candidat représentant le parti au pouvoir, le Rassemblement des Houphouëtistes pour la Démocratie et la Paix (RHDP), par ailleurs proche collaborateur du Président de la république, SEM Alassane OUATTARA, fortement soutenu par le Vice-président, Monsieur Kablan Duncan, Député de Grand-Bassam. Le second est Monsieur Jean Philippes EZALE, maire sortant et candidat du Parti Démocratique de Côte d'Ivoire (PDCI), parti d'opposition.
} 
période de tenue de l'Abissa qui devait consacrer le nouvel an N'zima avec la possibilité de l'exercice de la critique sociale ; occasion d'épuration des tares dans la gestion sociopolitique de la communauté par le roi (J.D. Agbroffi, 1996, p 79) verra un soulèvement social des composantes de la société N'zima qui se soldera par la «profanation » de la place de l'Abissa ainsi que de tous les symboles (T. K. Melèdje, 2018, p. 2). L'un des tenants anthropologiques et historiques des symboles demeure la procession des sept matriclans ${ }^{2}$ et leurs symboles alimentaires qui doivent être bénies par des prêtresses pour garantir l'abondance dans le royaume, le cas échéant, la famine sévirait. Quoique avertie des incidences de la rupture dans la pratique de l'Abissa sur la société N'zima, les jeunes en ont empêché les manifestations. Cette rupture flagrante (L. Baeck, 1958, p. 736) dans la tenue de l'élément culturel vital dans l'ordre multisectoriel du système N'zima sous la pression du corps social juvénil N'zima ouvre la réflexion sur son degré d'appropriation des valeurs communautaires et de légitimation de leurs actes (J. F. Guillaume, 2000, p. 115). Dès lors, il convient de s'interroger comme suit: comment les dissensions internes du peuple N'zima affectent-elles la pratique de l'Abissa et quelles en sont les conséquences ? Pour ce faire, l'objectif de cette recherche est d'analyser les incidences des dissensions internes du peuple N'zima sur la pratique de l'Abissa, ainsi que les conséquences qui en découlent.

\section{Matériels et Méthodes \\ 1.1. Matériels \\ 1.1.1 Champ géographique}

Située à 43 kilomètres à l'est d'Abidjan, la localité de Grand-Bassam est le chef-lieu du département de Grand-Bassam dans la région du SudComoé. Il est situé entre le $9^{\circ} 32$ de latitude nord limité par les Aowin, les Wassa et $6^{\circ} 29$ de longitude ouest limitée par les Ehotilé et les Essouma. Le sud de la ville est limité par l'Océan Atlantique et l'Est par les Fanti. La population de la ville de Grand-Bassam est estimée en 2000 à 73.772 d'habitants et 83576 habitants en 2010 vivant sur une superficie de $940,5 \mathrm{~km}^{2}$, soit une densité moyenne de 79 habitants par $\mathrm{Km}^{2}$.

La ville est essentiellement touristique même si elle bénéficie d'un climat de type attiéen, avec une pluviométrie abondante favorisant quelques activités agricoles caractérisées par le vivrier et les cocoteraies. Le commerce du fait de son attractivité touristique surtout avec la présence du cordon littoral et de la lagune Ebrié, la lagune Ouladine qui est un bras de lagune ébrié et du fleuve Comoé est dominé par la vente (bijou, pagne-kita, or, sel) ainsi que d'autres activités.

${ }^{2}$ Les sept matriclans N'zima sont : les Ndweafo, les Ezohile, les Nvavilè, les Adahonlè, les Alonwoba, les Azanwulè et les mafôlè 
Cette étude a eu lieu dans les espaces suivants : le quartier Impérial, le quartier France et enfin le village d'Azuretti. Le choix de ces sites d'étude ne s'est pas fait de façon fortuite mais obéit au principe d'objectivité. En effet, le choix de ces sites d'étude s'est opéré sous la base de l'enquête exploratoire du mois de Novembre 2018 qui a permis de constater la réticence des autochtones suite aux élections municipales observées à Grand-Bassam créant ainsi des discordes entre les acteurs et aux différents effets observés en termes de destruction surtout de profanation du site de l'Abissa.

Figure 1 : Localisation des zones d'étude avec les localités cibles de l'étude

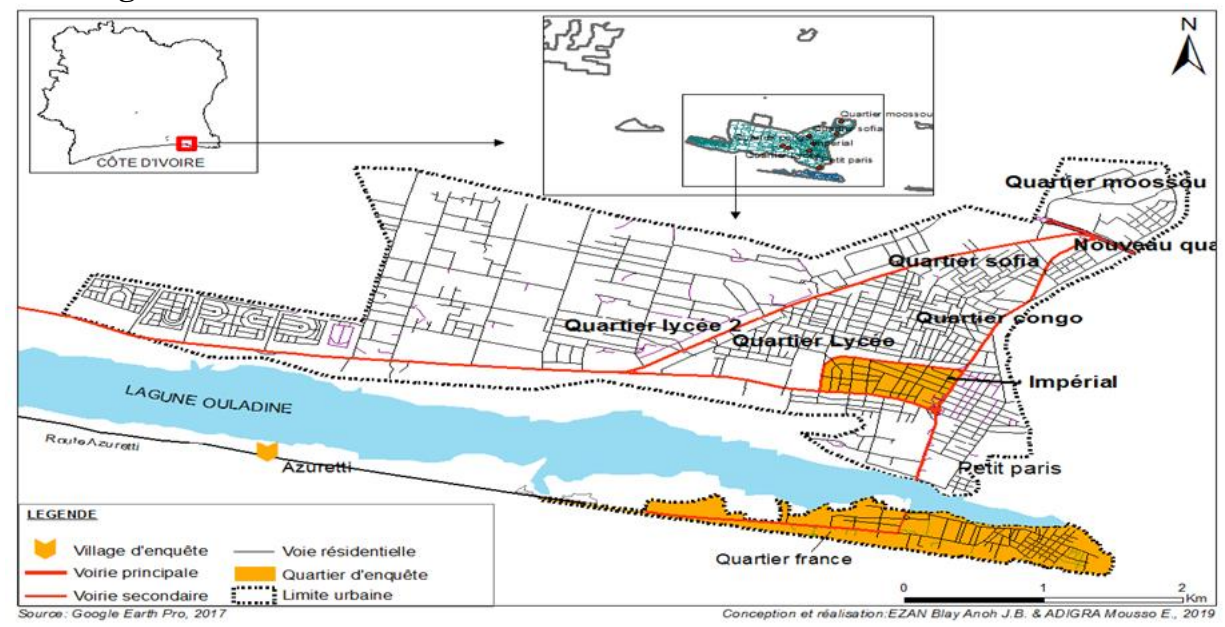

\subsubsection{Les techniques et outils d'enquête}

Pour la collecte des données, les techniques de l'entretien et de l'observation ont été utilisées. Ainsi, comme outils un guide d'entretien individuel, une grille d'observation, un dictaphone, un bloc-notes et un appareil photo ont servi à la collecte. La nature qualitative des informations à rechercher a conduit à s'orienter vers les informateurs clés dans la société N'zima susceptibles de renseigner sur les fondements de l'Abissa, ses principes mais également sur les motifs de la rupture observée dans sa pratique pour l'année 2018 ainsi que les incidences sur la société N'zima. Ces informations ont pu être livrées par les chefs traditionnels dont le secrétaire adjoint de la cour royale, le porte-canne du roi, les chefs des sept (7) familles qui compose la société N'zima notamment la famille $N$ 'vavilé (détentrice des us et coutumes N'zima Kotoko), le responsable des chansonniers (Ablamon ${ }^{3}$ et $\left.A n a z e^{4}\right)$ de l'Abissa ainsi que la délégation des Komians du royaume. Pour plus de précisions sur les violations observées dans la pratique de l'Abissa, le responsable communautaire de la tradition de l'Abissa fut également

\footnotetext{
${ }^{3}$ Nom des chansonniers de quartier France

${ }^{4}$ Nom des chansonniers du village d'Azuretti
} 
interviewé. Ainsi douze (12) leaders traditionnels ont-ils été interviewés. Dans la mesure où les jeunes ont été très actifs dans l'exacerbation de ces différends, les leaders de la jeunesse N'zima et des autres communautés, ainsi que les responsables communautaires, au nombre de dix (10) personnes ont pris part aux entretiens. Pour comprendre les tenants politiques de ces différends, le responsable local de la Commission Electorale Indépendante (CEI) et les directeurs de campagne des deux candidats aux prises lors des élections municipales ont pu être interrogés. Les échanges avec eux ont permis de comprendre les enjeux autour des résultats et les conflits communautaires qui en ont résulté. Au fur et à mesure des investigations de terrain, le constat du seuil de saturation d'informations a permis de mettre fin aux enquêtes qui se sont déroulées de Juin 2018 à Avril 2019. Ainsi, vingt-cinq (26) personnes ont été enquêtées.

\subsection{Méthodes d'analyse}

La lecture du contexte social étudié met en évidence une série d'acteurs que l'on peut qualifier de parties prenantes. Ainsi, la compréhension des actions de ces derniers à la suite du dépouillement fait des entretiens réalisés a mis à contribution la théorie des parties prenantes, la théorie des conflits de culture et de déviance des jeunes. L'approche ethnographique a aussi été mobilisée pour la description des situations rencontrées lors des enquêtes.

A la lumière de la théorie des parties prenantes (stakholder theory), les intérêts des différents acteurs notamment les jeunes, la notabilité voire la royauté, les Komian ${ }^{5}$ ainsi que les différentes familles constitutives de la société N'zima sont mises en corrélation. Ainsi, la mise en scène de chaque groupe d'acteur traduit une motivation (J.D. Rendtoff, M. BonnafousBoucher, 2014, p.58) qui sous-tend sa position dans l'arène des revendications pour les jeunes, le soutien au roi manifesté par certains notables, la frustration des chefs des matriclans et l'intrusion des tiers parties dans les manifestations observées. Dans cette même perspective « tout groupe ou individu qui peut affecter ou qui peut être affecté par la réalisation des objectifs d'une organisation», (op cit., p. 76) entendu ici comme effets de la position politique du roi se retrouve partie prenante. De cette sorte, la théorie des parties prenantes révèle des actions anticonformistes des locaux au sujet de leur tradition mise en mal.

\footnotetext{
${ }^{5}$ Les Komian sont les prêtresses traditionnelles en charge de la préparation spirituelle de l'Abissa. Leur rôle s'apparente à celui de gardiennes du temple, ici dans la circonscription de l'espace-temps et de l'espace géographique de la cérémonie à travers les rituels d'exorcisme effectués avant le démarrage, lors des festivités, la conjuration des sorts à la sortie de l'Adonvêlê (tambour sacré) qui marque le temps de parade du roi sur la place de la l'Abissa. Leur sortie est gage de sûreté.
} 
La situation à travers la théorie des conflits de culture est comprise dans un sens de généralisation voire d'intervention de groupes ethnolinguistiques non n'zima dans les manifestations menées en l'encontre du roi dont la résultante a été la violation du sacré avec la profanation de l'Abissa. Il se perçoit des germes de délinquance liés aux confrontations culturelles des acteurs en situation (F. Brion, F. Tulkens, 1998, p. 243). Cellesci s'illustrent dans le cas d'espèce par l'implication de communautés non autochtones notamment malinké dans un différend communautaire lié à la pratique de l'Abissa. Cette intrusion extérieure a entrainé le mécontentement et la réaction des autochtones n'zima. Outre cette externalité, peut être relevée celle d'une mainmise des pouvoirs publics de plus en plus fréquente sur cette valeur traditionnelle du peuple N'zima. Ainsi, cette ingérence du politique dans le culturel donne libre cours au conflit idéologique situant un écart entre les décisions émanant du politique et les modes de pensées et pratiques traditionnelles.

\section{2- Résultats}

Ces résultats relèvent les données empiriques de terrain émanant des enquêtes.

\subsection{Facteurs d'émergence des dissensions internes dans la société N'Zima}

Plusieurs facteurs ont fait leur apparition dans l'arène sociale N'zima et ont conduit à des dissensions internes.

\subsubsection{L'intrusion de tiers dans la pratique de l'Abissa comme acte de sa désacralisation}

L'Abissa se présente comme une institution sacrée héritée des ancêtres et qui doit garder ce caractère comme l'attestent les propos d'un notable.

«Les gens voient l'Abissa comme quelque chose de trop simple. Or, quand nos parents recevaient l'Abissa de l'ancêtre "Afoantchè », c'était dans le secret. Donc tout ce qui concerne l'Abissa est sacré, ce n'est pas tout le monde qui fait et qui voit, cette valeur doit au moins demeurer pour être transmise à nos enfants »

L'Abissa en effet est une pratique cultuelle et culturelle célébrée par les ancêtres depuis le Ghana et ne concernait en réalité que les acteurs clés issus de la configuration communautaire N'Zima. La valeur, ainsi que le respect au bon fonctionnement de ce forum traditionnel lui conférait son caractère ésotérique. Les géniteurs de cette pratique limitaient en effet cette danse aux individus ayant le droit d'y participer puisqu'elle se faisait selon 
une convention spirituelle. C'est ce que nous révèle la responsable des prêtresses (Kômian) :

«Dans le temps, il fallait être un sorcier, un clairvoyant, un revenant, un féticheur pour danser l'Abissa. C'est comme le Dipri chez nos frères Abidji de Sikensi ou le Fokouê des Abouré. Est-ce que tu vois les dioulas, les mossies et autres ethnies venir danser aussi ? C'est comme ça qu'on dansait l'Abissa ; ce n'est pas l'Abissa de maintenant qui est dans la main des dioula, les politiciens qui viennent acheter ce qui appartient aux N'Zima. » N.B, responsable des Kômian de Grand Bassam.

L'allusion faite au Fokoué ${ }^{6}$ chez les abouré et le Dipri des abidji traduit le caractère sacré et ésotérique de l'Abissa qui n'était réservé à la pratique qu'aux seuls initiés mais bien plus aux initiés N'zima qui seuls en comprennent le langage, les sens et les symboles.

L'intrusion des allochtones dans la gestion des pratiques coutumières des N'zima est perçue par une frange de la population N'zima comme une déchéance, une profanation du sacré et crée un désaccord au sein de ladite communauté entre conservateurs et réformistes. Il s'observe un désordre comme le traduit le chef de la famille N'vavilé :

«Ces allochtones ne disposant d'aucune valeur éthique mettent en mal la célébration de l'Abissa dans la mesure où certains acteurs se font appelés dioula Kôtôkô se réclamant des mêmes droits que les autochtones. On pourrait bien se poser cette question de savoir si cette pratique est pour les dioula ou le peuple N'Zima? De plus, il y a beaucoup d'interdits autour de la pratique de l'Abissa que tout le monde ne connaît. C'est pour cela que toutes les phases de l'Abissa ne sont pas portées au regard de tous »,

Il poursuit en indiquant les sensibilités et autres spécificités de l'Abissa qui semblent aujourd'hui foulées au pied par le regain de modernisation de cette institution

«Les étapes de l'Abissa étaient strictes car en plus de l'organisation qui concernait uniquement deux entités à savoir l'Ablamon et l'Anazè, il y avait aussi des formalités communautaires qui étaient exigées, notamment la provenance de l'individu qui vient prendre part à la fête, son appartenance familiale etc. Cependant, les étrangers se contentaient de

\footnotetext{
${ }^{6}$ Le Fokoué chez les abouré est une danse ésotérique réservée qu'aux initiés membres d'une classe d'âge mieux d'une génération. Il est avec le chef guerrier «Sanflan » les symboles réservés qu'à ceux qui en comprennent le langage et sont habilités à en danser.
} 
l'observation de la danse qu'à la sortie officielle de l'Abissa suivie de la parade du roi devant le peuple »

Lui emboitant le pas, l'un des acteurs clés des chansonniers de la critique sociale pendant la sortie de l'Abissa devant le roi s'exprime comme suit :

«L'Abissa perd sa valeur culturelle car elle est influencée par l'évolution de la modernité. Si aujourd'hui le calendrier N'zima doit tenir compte du regard de l'Etat avant de célébrer sa fête annuelle, c'est que les institutions traditionnelles perdent leur valeur. On ne peut mélanger la tradition avec la politique ni la modernité et c'est ce qui provoque les conflits d'ailleurs. Parce que le fait qu'on entraine les leaders communautaires à vocation apolitique à la politique créé nécessairement des points de divergences. » E.A, membre à la notabilité de Grand Bassam.

De son point de vue, il y a des attributs qui doivent impérativement demeurer dans l'ordre du sacré, de l'interdit quelle que soit l'évolution de la société.

«Il est vrai que Grand-bassam est patrimoine historique de l'Unesco mais ça doit rester juste sur le plan politique afin que nos valeurs traditionnelles ne soient pas bafouées »

Cette lecture est partagée par le chef de la famille Ezohilé, l'une des sept familles claniques N'zima.

L'on dénote de tout ce qui précède que l'ouverture faite et qui autorise une intrusion des non N'zima dans la sphère de la pratique de l'Abissa est de nature à créer des incompréhensions internes. Une frange des natifs N'zima conçoit la participation à toutes les étapes de l'Abissa par des populations non N'zima comme un acte de violation de la sacralité de l'institution. C'est par ailleurs une source de fragilisation des fondamentaux même de la société n'zima.

Par ailleurs, à l'analyse des propos plus haut, une explication permet de comprendre ces griefs émis. L'Abissa depuis son origine se pratique dans le mois de septembre (mois qui marque le nouvel an dans le calendrier N'zima) et qui requiert une purification du peuple, une critique de la gouvernance des tenants du pouvoir notamment le roi et la conjuration des mauvais sorts par les Komians. Celles-ci constituent la garde spirituelle du peuple. Par leurs actions, et la définition des actions futures de développement communautaire, la société dans une perspective progressiste. Cependant, cette année 2018, du fait des élections, le gouvernement a instruit le ministre de la culture de reporter 
la célébration après les élections, au mois de novembre. Toutes choses qui ne rencontrent pas l'assentiment de tous comme l'attestent les propos du secrétaire adjoint du roi et ceux de la prêtresse principale :

«C'est trop difficile avec le gouvernement qui veut tout décider maintenant dans la célébration de l'Abissa, parce qu'on voit maintenant comment gagner l'argent avec. Hors, les génies qui nous conduisent ne sont pas à vendre. En plus, il y a le calendrier spirituel à respecter. Agir hors du temps marqué par les génies pour accepter les offrandes qu'on leur fait est un sacrilège »

A ce facteur déjà source de clivages sociaux internes s'adjoint celui qui suit et qui est en rapport aux échanges des élections municipales du 13 octobre 2018.

\subsubsection{Dissensions internes liées aux résultats des municipales d'octobre 2018}

Les dissensions internes liées aux résultats des élections municipales d'octobre 2018 trouvent leur fondement dans ce qu'il convient d'appeler selon le regard des enquêteurs « la trahison du roi » comme relevés dans les propos qui suivent.

«Un roi doit savoir dissimuler sa position politique quand il gère un peuple avec plusieurs sensibilités politiques (...). Ce que le roi a fait est une trahison de son peuple. Comment face à deux candidats, il choisit de soutenir ouvertement jusqu'à affirmer avant même les conclusions des délibérations des moyens de recours saisis par un des candidats la victoire de l'adversaire du candidat n'zima» $\mathrm{O}$. A, responsable de jeunesse N'zima du quartier France

Abondant dans le même sens, l'un des notables influent de la cour royale estime :

«Nous sommes tous restés perplexes face à la sortie du roi sans consulter les sages qui l'entourent. Il est certes le Président de la Chambre des Rois et Chefs traditionnels de la Côte d'Ivoire, on sait comment il y est parvenu ${ }^{7}$, mais quand il se retrouve dans la société n'zima, il doit comprendre qu'il est le reflet du peuple et le modèle qui crée la cohésion et non le représentant et le porte-voix des ambitions politiques au point de décider et

${ }^{7}$ Ce sous-entendu fait allusion aux tractations politiques pour que le roi n'zima soit nommé par le Président Alassane Ouattara à ce poste et les implications en termes d'exécution de la volonté de ce dernier surtout au plan politique. 
de proclamer des résultats (.....). C'est un manquement criant à son devoir de chef de surcroit traditionnel incarnant les valeurs d'impartialité mais surtout de maintien de la cohésion de sa communauté. Il a réussi à créer la scission même parmi les notables que nous sommes. Il y a toujours des bénis oui-oui qui le soutiennent dans ces démarches hasardeuses. » M.O, Porte-parole adjoint de la cour royale

Ces deux interventions traduisent le désaccord entre le roi et une frange de sa population sur son intervention lors de la proclamation des résultats de l'élection municipale à Grand-Bassam. En effet, à l'issue du premier tour, les résultats ont été contestés par le candidat du parti démocratique de Côte d'Ivoire (PDCI) déclaré perdant au profit du candidat du Rassemblement des Houphouëtistes pour la Démocratie et la Paix (RHDP) au pouvoir depuis 2011 en Côte d'Ivoire. Par ailleurs, le premier cité est ressortissant de la communauté N'zima tandis que le second est natif Abouré. Ces deux groupes ethniques qui partagent la localité de Grand-Bassam s'en disputent la propriété terrienne, ainsi que l'hégémonie politique. Dès lors, l'on observe un glissement de ce qui était un différend entre N'zima sur le terrain politique comme le relève le président local de la CEI en ces termes :

«A regarder la situation de près, ce n'est pas vraiment les résultats des élections qui sont contestés par les parents N'zima, c'est une lutte d'hégémonie entre abouré et N'zima pour le contrôle de Grand-Bassam en tout cas du point de vue politique. Le roi a joué tout simplement de raison, pour selon moi maintenir un équilibre en appelant à la cohésion et s'en tenir aux résultats proclamés par nos services »

Cette lecture est confortée par cette récrimination émise par le chef du quartier France de Grand-Bassam :

« Comme le roi s'associe à des imposteurs pour nous voler notre victoire alors qu'il parte où qu'il démissionne afin que notre digne fils Ezaley soit notre roi. Et s'il ne veut pas perdre cette position qu'il restaure la justice afin qu'Ezaley soit blanchi $^{8} \gg($ K.A, chef de quartier France de Grand-Bassam).

La même lecture est faite par le président des jeunes N'zima : «On nous a volé notre victoire et ça, c'est la faute au roi qui a soutenu cette forfaiture. Il est vendu et avec ça, il veut venir

\footnotetext{
${ }^{8}$ Implicitement, qu'Ezalé soit déclaré vainqueur au détriment de son adversaire.
} 
faire Abissa de nos ancêtres. Ce n'est ni aujourd'hui, ni demain » H.M, président des jeunes N'zima

Ces velléités de suspicion s'intensifient à en croire ce qui suit : «Depuis quand un roi livre sa communauté pour de l'argent? Il bafoue les valeurs de notre tradition, il n'est pas digne d'être notre leader. Et comme il nous impose un candidat, on ne dansera pas l'Abissa. Que lui et son candidat à viennent danser! ». A. K, Adjoint des chansonniers de Grand-Bassam (Ablamon)

Ces propos traduisent le malaise social qui est survenu dans la société N'zima à l'issue des élections municipales et qui entachent la pratique de l'Abissa. Ainsi le contexte politique devient-il l'un des facteurs des controverses qui ont eu un impact négatif sur la tenue de l'Abissa l'année 2018, avec une rupture de la confiance du peuple N'zima en son souverain au point de demander sa destitution.

\subsection{Incidences des dissensions sur les principes fondamentaux l'Abissa et la société N'Zima}

Les différents facteurs ont induit des incidences sur les principes fondamentaux de l'Abissa et la société N'zima.

\subsubsection{La profanation de l'institution Abissa et ses incidences}

L'une des incidences majeures se traduit par la destruction des tribunes de l'Abissa, la profanation de l'espace public qui représente la forêt sacrée du peuple, la destruction des bâches et les dégâts sur l'habitat du garant communautaire comme le traduisent les images ci-dessous :

Figure 2 : Destruction des installations pour les festivités de l'Abissa

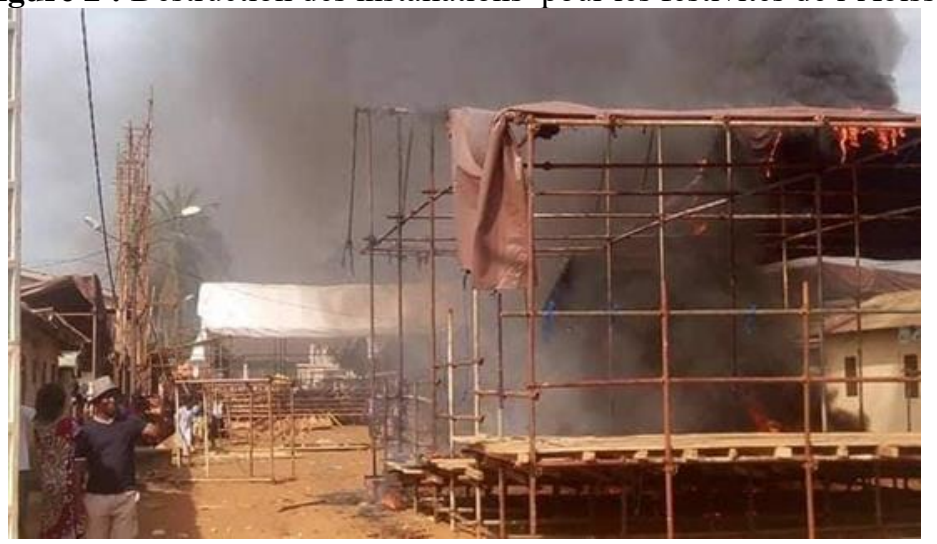

Source : Images d'enquêtes, avril 2019 
Figure 3 : Désacralisation des tambours sacrés (Adonvlê) de l'Abissa

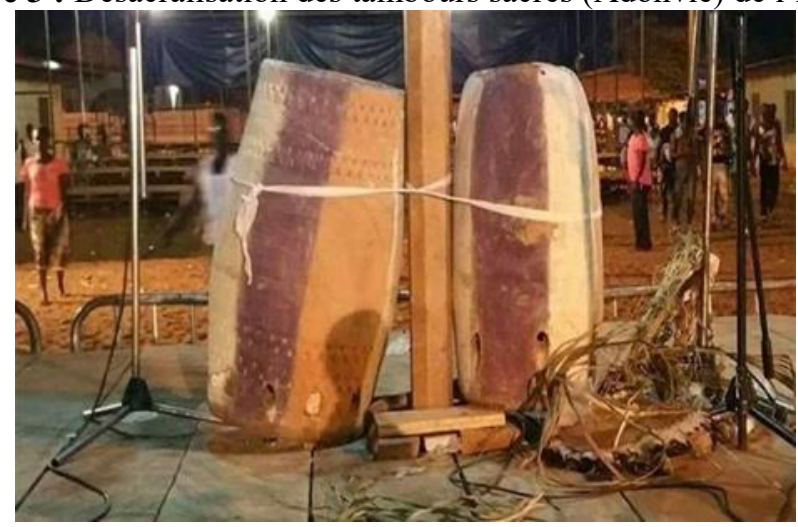

Source : Images d'enquêtes, avril 2019

A ces images, s'adjoignent les propos d'un des membres du comité d'organisation :

«C'est parce qu'il voit qu'on a monté les bâches, le podium et la place pour les tambours qu'ils espèrent qu'on va danser l'Abissa ; donc on a tout brulé, tout démonté pour montrer aux yeux de tout le monde que l'Abissa est pour nous les N'Zima pas pour d'autres ethnies et tant que la vérité n'est pas prise en compte, on manifestera toujours cette colère. On ne fête pas dans l'injustice, dans la tricherie, dans l'hypocrisie. » (K.M, membre comité d'organisation de l'Abissa).

Ces images et ses propos traduisent d'une part le mécontentement du peuple N'zima et induisent les incidences sur la société N'zima. Ce péril occasionne du point de vue des tenants des dimensions magico-religieux de l'abissa une irritation des génies tutélaires et une perturbation des énergies cosmiques en faveur du peuple N'zima comme relevés ci-dessous :

«L'Abissa pour ne pas dire, la sortie de l'Abissa symbolise le nouvel an en pays N'zima. C'est en cette occasion que les mannes des ancêtres fondateurs y sont invoquées. C'est également en cette occasion que la société toute entière procède à faire sa mue par la critique sociale, les rituels de purification et de conjuration des mauvais sorts, l'aplanissement des différends et autres crises en vue de la réconciliation sociale entre tous les fils et filles du peuple N'zima. Ces actions augurent de meilleurs lendemains pour tous. Cette année sera terrible même parce ce que ce qui constitue le socle de la société N'zima est bafouée. L'une des conséquences peut surtout être les inondations parce que le peuple N'zima est très attaché à l'eau et les rituels servent aussi à rendre les génies 
cléments et éloigner ce type de fléau. Nous sommes en danger mon frère et tout ça à cause de la soif des gens mêmes qui nous gouvernent. Le plus grave, c'est le fait que si l'Abissa ne fait pas sa sortie cette année, nous sommes interdits pour cinq ans sans pratique. Les conséquences seront incalculables. On est donc obligé de procédé au moins à la purification de la terre » Y.M, chef de la famille N'vavilé (détentrice des us et coutumes N'zima)

Ces propos de l'acteur clé des pratiques ancestrales traduisent tout le malaise auquel s'expose la communauté N'zima du fait des crises internes et de la non tenue de l'Abissa en cette année 2018. Pour le responsable communautaire la tradition de l'Abissa, il faut palier au plus urgent et au plus vital :

«Il est vrai que compte tenu de tout ce qui s'est passé, cette année, l'Abissa ne se pratiquera pas comme les autres années. Il y a eu tellement de sacrilèges. D'un commun accord avec les prêtresses qui ont consulté les mannes des ancêtres et nos génies tutélaires, elles vont juste procéder à la purification de la place de l'Abissa pour ne pas freiner la célébration pendant cinq (5) ans. Les conséquences seront incalculables. Cette cérémonie tiendra sur une journée voire même deux (2) heures de temps. Cela adoucirait les génies et préservera notre société ». B. N, Responsable communautaire de la tradition de l'Abissa

Ces dispositions prévenantes n'occultent nullement la forte fragilisation des rapports sociaux et l'émergence des conflits communautaires.

\subsubsection{Fragilisation des rapports sociaux communautaires et émergence des conflits}

Ce qui au départ n'était qu'un conflit communautaire N'zima va basculer dans l'intercommunautaire comme le soulignent certains enquêtés : «C'est tellement étonnant la tournure que les choses ont prises. Nous avons tous observé surtout au quartier France le saccagement de la place de l'Abissa qui est notre bois sacré à nous, comment les jeunes surtout sont allés huer le roi au palais au cours d'une de ses réceptions, mais quand les affrontements ont commencé à se généraliser entre jeunes N'zima et les autres jeunes notamment malinké que l'on appelle communément Dioula du quartier Phare, vraiment on a rien compris. » A.J, Conseiller Municipal à la mairie de Grand-Bassam. 
La compréhension de cet étonnement se trouve dans les explications du Président des Jeunes de Grand-Bassam :

«Pendant que nous N'zima cherchons à régler nos problèmes entre nous, nos parents Dioula, on ne sait trop pourquoi se lèvent et exigent eux aussi que l'Abissa se tienne. Ils font même manifestation de soutien au ministre de la Culture qui lui également tient forcément à ce que l'Abissa ait lieu cette année parce qu'il y aurait des engagements pris par nous ne savons qui. Mais l'Abissa, c'est une affaire de N'zima, seuls les N'zima peuvent décider de le faire ou pas. C'est surtout ce qui a énervé les jeunes qui ont fait une riposte avec même des blessés et l'incendie de la mairie» K.S, Président des jeunes N'zima de Grand-Bassam

Ces propos sont corroborés par les images ci-dessous qui traduisent les violences communautaires :

Figure 3 : Incendie de la mairie de Grd-Bassam pendant les manifestations

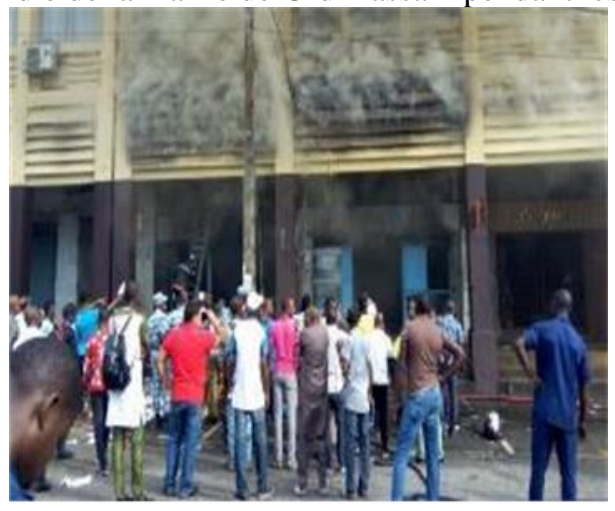

Source : Images d'enquêtes, Avril 2019

Figure 4 : Marche de protestation des femmes et des jeunes

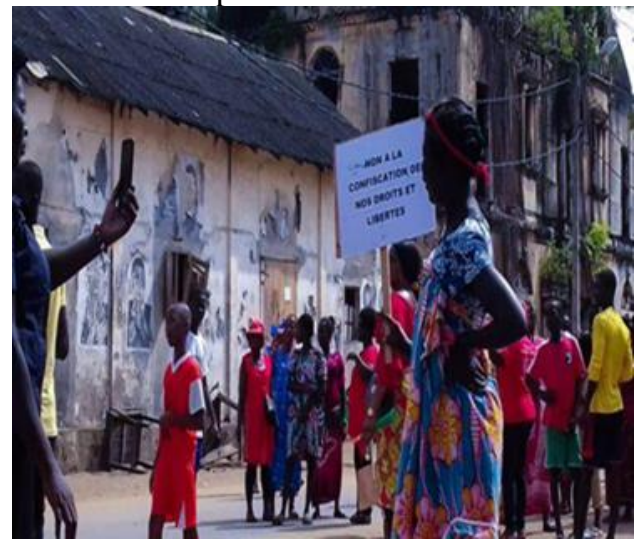

Source : Images d'enquêtes, Avril 2018 
Figure 5 : Un jeune blessé lors des manifestations communautaires

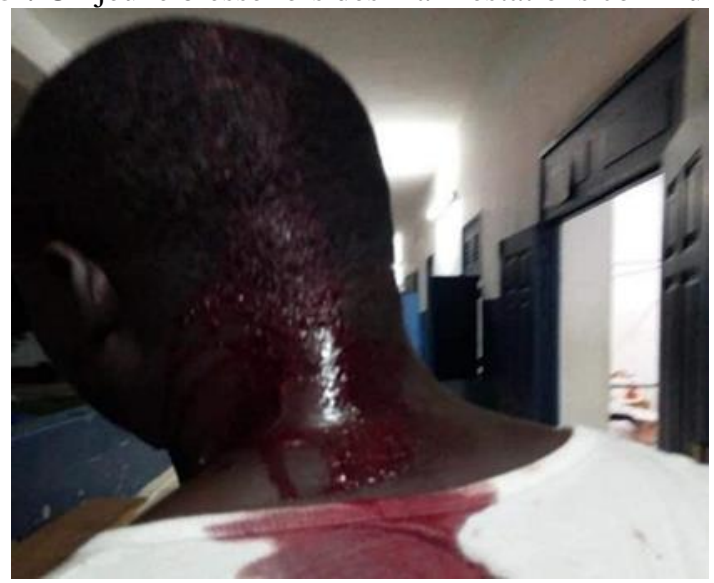

Source : Images d'enquêtes, Avril 2019

Les images traduisent l'ampleur des violences intercommunautaires qui mettent aux prises les différentes parties prenantes et leur logique d'action. A cet effet, l'un des responsables de la communauté malinké interrogé soutient :

«Je ne sais pas trop ce qui pousse nos jeunes à aller même s'immiscer dans cette affaire. Ils voient une affaire de RHDP et ça crée des tensions interethniques, parce qu'on voit en RHDP dioula. Au fond, c'est politique tout ça là, on met tout au compte de la politique et ça n'arrange personne sinon que des destructions »

La question politique comme point d'ancrage des violences observées se confirme avec cet enquêté et met les jeunes au cœur des violences observées.

\section{4- $\quad$ Analyse et discussion}

Les résultats de cette recherche mettent en évidence l'intrusion des acteurs exogènes à la communauté N'zima qui viennent perturber l'organisation sociale existante. Il s'agit notamment des pouvoirs publics dont les intérêts surpassent ceux du peuple N'zima pour qui la pratique de l'Abissa reste et demeure de l'ordre traditionnel. Pour les autorités politiques et administratives par contre, l'institution est une vitrine de promotion nationale. Ces logiques divergentes plongent au cœur d'une instabilité institutionnelle dans laquelle l'influence des cadres politiques et leurs courses au positionnement et au leadership local, l'intrusion des luttes pour le pouvoir à l'échelle nationale dans l'arène villageoise et des questions générationnelles sont dépeintes. Cette situation au regard de (L. Baeck, 1958, p.734) fait perdre 
l'autonomie politique des sociétés traditionnelles ainsi que leur influence sur le système social. Abordant la question de cette même perte d'autonomie dans le sens contraire, (J-F. Bayard, 1989, p. 296) note dans son ouvrage «L'Etat en Afrique. La politique du ventre » que :

«la responsabilité de la déstructuration des micros états traditionnels relève de l'avidité des gouvernants même traditionnels qui se laissent phagocytés par la machine politique d'Etat $\gg$.

Toutes choses donc qui mettent en relief les aventures ambiguës du pouvoir traditionnel à la politique (V. Foucher, E. Smith, 2011, p. 36) dans la mesure où les registres d'expression et de gestion ici en œuvre dans le contexte moderne contrastent à bien des égards avec les cadres traditionnels (G. Balandier, 1967, p. 42). Cette lecture se justifie au regard des griefs portés par le peuple N'zima contre le roi à qui il est reproché un parti pris dans une élection. Certains dans son peuple le qualifient de «vendu », tandis que d'autres voient en son choix un symbole de conciliation dans un climat morose.

Dans cet environnement, les griefs portés par une frange de la communauté N'zima contre le roi jusqu'à créer une rupture dans la pratique annuelle de l'Abissa est assez évocateur. Et pour cause, cette célébration est l'occasion annuelle unique de retrouvailles des sept matriclans (Aboussouan) afin de faire un bilan global d'exercice des pouvoirs et des actions communautaires au regard de la constitution locale. Par ailleurs, le rôle joué par les jeunes dans la contestation des résultats des élections et les manifestations allant des destructions voire à la profanation de l'Abissa aux affrontements dépasse ce que (F. Brion et F. Tulkens, 1998, p. 248) interrogeant les conflits de culture et délinquance qualifient «d'incompatibilité de perceptions liées à une lutte de reconnaissance et de positionnement social dans une arène tenue par les aînés sociaux ». Dans le cadre de cette étude, le glissement des dissensions internes au peuple N'zima sur le terrain politique a été le terreau de développement de nombreuses controverses internes et des conflits intercommunautaires traduisant ainsi la fragilité des mécanismes traditionnels de transformations des conflits. Cette situation au regard de (B. M. Rukakiza et al., 2015) dans leur ouvrage « L'État africain et les mécanismes culturels traditionnels de transformation des conflits » abordent les dissensions culturelles sous une approche qui montre que les controverses liées à la culture des acteurs sont parfois provoquées par l'Etat qui s'ingère dans les rapports socio-culturels de ces derniers. Aussi montrent-ils à travers l'exemple du peuple congolais, l'échec des reformes de l'Etat visant à créer l'entente entre les acteurs. Cet échec est lié à l'importation 
des méthodes de transformation des conflits nationaux qui ne tiennent pas compte des particularités provinciales et locales.

Ce contexte qui laisse entrevoir des compétitions et pour le pouvoir et pour l'hégémonie générationnelle au détriment de l'Abissa a des conséquences sur l'équilibre de la société N'zima. Les données recueillies établissent deux ordres; les conséquences magicoreligieuses et les conséquences sociales. A ce propos, la violation des interdits prônés dans l'Abissa notamment la violence matérialisée par l'absence de la critique sociale et la purification du tambour sacré constitue selon (D.J Agbroffi, 1996, p. ; R.K. Allou, 2011, p. 112) un vecteur d'assombrissement de l'année pour le peuple N'zima tandis que pour (F.B. Eboussou, 1976, p. 186 ), c'est un marqueur de renoncement à son identité, notamment ethnique. A la différence de ces deux auteurs, l'Abbé (A. Bazié, 2007) pense que la pénétration des religions révélées notamment la religion chrétienne dans les sociétés traditionnelles devient le catalyseur à une défiance de plus en plus fréquente des croyances et autres rites traditionnels qui en perdent leur valeur de vie. En fin de compte l'hétérogénéisation de la société N'zima semble favoriser une dislocation de la culture de base qu'est l'Abissa.

\section{Conclusion}

En définitive, nous retenons à l'analyse et à la discussion des résultats de cette étude que la pratique de l'Abissa au cours de l'année 2018 a connu une rupture du fait de facteurs exogènes à la société N'zima. Parmi ceux-ci, l'on peut retenir comme premier facteur l'immixtion de tierces parties dans la pratique de l'Abissa foulant ainsi au pied les principes culturels de base de l'institution. Lesquels principes réservent les seuls droits aux N'zima dans l'ordre des valeurs d'indiquer les périodes propices aux manifestations de l'Abissa. Toute intervention de quelque nature que ce soit constitue une violation des prérogatives ancestrales du peuple. Le second est lié à la position ambiguë des leaders communautaires notamment du garant de la tradition dans le jeu politique en l'occurrence les élections municipales. Le roi des N'zima a effet pris position en faveur de l'un des candidats notamment celui du Rassemblement des Huophouétistes pour la Démocratie et la Paix (RHDP). Toute chose qui n'a pas été approuvé autant par certains membres de la cour royale et une frange importance de la société N'zima en l'occurrence la jeunesse. Ces mécontentements ont conduit à des manifestations de contestation contre d'une part les intrusions exogènes dans l'ordre décisionnel de la pratique de l'Abissa et le parti pris du roi. Ces manifestations ont occasionné des affrontements intercommunautaires sur fond de politique et ont concouru à la rupture constatée dans la pratique de l'Abissa.

Cette rupture, la première du genre, non liée à une contingence naturelle n'est pas sans conséquence pour le peuple N'zima. La valeur magico- 
religieuse de l'institution et sa portée spirituelle bafouée, le peuple s'expose au courroux des génies tutélaires. Peuples lacustres, l'un des dangers majeurs reste les inondations qui sont dans la perception traditionnelle, une punition des dieux pour non observance de leurs principes. Ainsi, le processus d'apparition des dissensions internes dans la société N'Zima révélé par les données de la recherche ont montré que la désacralisation de l'Abissa et l'ingérence du politique dans la gestion traditionnelle ont été des facteurs clés de déclenchement des clivages dans la société N'Zima. Il se pose ainsi la problématique de la survivance des institutions rationnelles dans un contexte moderne à multiples enjeux.

\section{References:}

1. AGBROFFI Diamoi Joachim, 1996, L'Abyssa et l'ordre social et politique chez les nzema. abidjan, cocody, Côte d'Ivoire, Thèse de Doctorat de $3^{\text {ème }}$ Cycle, $627 \mathrm{p}$

2. ALLOU Kouamé Réné, 2011. «Les sept matriclans et la tradition alimentaire chez les nzéma: essais d'interprétations historiques. » Revue Hist-Archéo. AFR, Godo Godo, N²1, pp 108-115

3. ALLOU Kouamé Réné, 2013, Les Nzema : une peuple akan de Côte d'Ivoire, L'Harmattan, Paris, $238 \mathrm{p}$.

4. BAECK Louis, 1958, « Le changement social en Afrique centrale » in Bulletin de l'Institut de recherches Economiques et Sociales, $\mathrm{N}^{\circ} 8$, déc, pp 729-768

5. BALANDIER Georges, 1967, Anthropologie Politique, PUF, France, $237 \mathrm{p}$

6. BAYART Jean - François, 1989, L'Etat en Afrique. La politique du ventre, Fayard, Paris, $439 \mathrm{p}$

7. BAZIE Alexandre, 2007. Croyances religieuses et valeur de vie, Communication au Congrès International de Bioéthique Octobre Ouaga (BF), $17 \mathrm{p}$

8. BENN Eifert, EDWERD Miguel et POSNER N. Daniel, 2010, «Political competition and Ethnic Identification in Africa » American journal of Political Science, (54) (2), 494-510

9. BRION Fabienne et TULKENS Françoise, 1998. Conflit de culture et délinquance. Interroger l'évidence, Déviance et Société, Vol 22, Nㅜ 3, pp 235-262

10. EBOUSSOU BOULAGA Francis, 1976. L’identité Négro-africaine in Présence africaine, Essai. Paris, $239 \mathrm{p}$

11. PAULME Denise, 1960. «Structures sociales traditionnelles en Afrique Noire », Cahiers d'Etudes africaines, $\mathrm{Vol}^{\circ}{ }^{\circ}$, pp 15 - 27 
12. FOUCHER Vincent et SMITH Etienne, 2011. Les aventures ambigües $\mathrm{du}$ pouvoir traditionnel dans l'Afrique contemporaine, Revue Internationale et Stratégique, $\mathrm{N}^{\circ} 8$, pp 30-43

13. GUEYE Mamadou, 1983, «Transformations sociales et implications culturelles », Ethiopiques $\mathrm{N}^{\circ} 34 \& 35, \mathrm{Vol} 1, \mathrm{~N}^{\circ} 3$ et 4

14. GUILLAUME Jean François, 2000, «Des jeunes face aux institutions », Lien social et politique, $\mathrm{n}^{\circ} 43$, pp 113-120

15. KOUAMÉ Louis Abrima, S, D, Histoire du peuplement des N'Zima (apolloniens) en côte d'ivoire, $23 \mathrm{p}$

16. MELEDJE Tresore Kouamé, 2018. Abissa 2018: «La cérémonie ne tiendra que deux heures de temps », LINFODROME, Soir Info du $07 / 11 / 2018$.

17. N'DOLI Didier, 2014. Grand-Bassam, métropole médiévale des N'zima, Editions du CERAP, Abidjan, $416 \mathrm{p}$

18. RENDTOFF Dahi Jacob et BONNAFOUS-BOUCHER Maria, 2014. Théorie des parties prenantes, la Découverte, Paris, $125 \mathrm{p}$

19. RUKAKIZA Muchukiwa Bosco, CIMNESA Bishweka Antoine, MASONGA Kapapa Camille, 2015. L'Etat africain et les mécanismes de transformation des conflits, Globethics.net Publications, $95 \mathrm{p}$ 